\title{
Design, Manufacturing and characterization of integrated inductors with magnetic layers for DC-DC converter
}

\author{
Dagal Yaya $^{1}$, Désiré Allassem ${ }^{1}$, Mahamoud Youssouf ${ }^{2}$, Ali Siblini ${ }^{1}$, \\ Jean Pierre Chatelon ${ }^{1}$ and Jean Jacques Rousseau ${ }^{1}$ \\ ${ }^{1}$ Université de Lyon, F-42023, Saint Etienne, France; Université de Saint Etienne, Jean Monnet, F-42023, Saint Etienne, \\ France; LT2C, F-42023, Saint Etienne, France. \\ ${ }^{2}$ IUSTA BP 6033 N'Djamena TCHAD \\ *corresponding author: siblini@univ-st-etienne.fr
}

\begin{abstract}
This paper presents the conception, fabrication and characterization of integrated inductors containing magnetic layers. A novel approach has been used to perform planar magnetic devices by using commercial ferrite substrate. In this paper Yttrium Iron Garnet is used as magnetic material due to the frequency range of the fabricated inductors (up to some hundred $\mathrm{MHz}$ ). For other application frequency ranges this approach is suitable, only the magnetic material has to be changed. We require different steps of microtechnologies: preparation of glass and ferrite substrates, RF sputtering, photolithography, etching and finally electroplating techniques for copper and gold films. The geometrical magnitudes are determined by using HFSS simulator software. The measurements performed at low and high frequencies (up to $1 \mathrm{GHz}$ ) permit to verify the correlation between experiment and simulation results. The inductance of the manufactured spiral inductor is about 200 $\mathrm{nH}$ and it is constant from low frequency up to $0.9 \mathrm{GHz}$.
\end{abstract}

\section{Introduction}

Considerable research has been recently devoted to the miniaturization of electronic components for the fabrication of high consumption portable devices (cellular phones, computers, cameras, avionics, motoring...) For such applications passive components are problematic due to their sizes. For example, in a typical amplifier MMIC (Monolithic Microwave Integrated Circuits), up to $80 \%$ of the area is occupied by chip inductors [1-3]. To overcome this problem many authors use magnetic materials in order to reduce inductor sizes. Different kinds of magnetic material are used such as ferromagnetic material, ferrite or sol-gel film doped with ferrite particles [4-6]. Ferromagnetic materials exhibit usually a low resistivity and have to be used either at low frequency or with very low thickness. Ferrite sintering requires very high temperatures and this approach is not really suitable for the fabrication of integrated circuits. Sol-gel films doped with ferrite particles is a low temperature process but lead to weak magnetic material concentrations.
Thus, to reach this purpose, we have to conceive outstanding miniaturized components capable to work at higher and higher frequencies.

For this reason, our laboratory is committed to the development of high frequency passive components in order to reduce their size and to increase their performance by using appropriate magnetic materials. A plate Yttrium Iron Garnet: $\mathrm{Y}_{3} \mathrm{Fe}_{5} \mathrm{O}_{12}$ (YIG) is used as magnetic material and copper as conductive layer. Our purpose is to manufacture integrated inductors based on thick magnetic layers for power converters applications. To conceive these inductors, a High Frequency Structure Simulation software (HFSS) has been used. The micro-manufacturing steps (Micromachining, RF sputtering, photolithography ...) will be described. And finally a comparison between the simulation and measurement results as a function of frequency and thickness of the magnetic core will be presented.

\section{Design of the spiral inductor}

We have used HFSS software for the conception and the simulation of the integrated spiral inductor. Figure 1 shows the design used for the simulation. The simulated structure consists of a glass substrate of thickness $E_{\text {sub }}$ used to improve the mechanical holding of the component, a magnetic thick layer of thickness $E_{\mathrm{YIG}}$, and a spiral copper film of thickness $e$ forming a coil of $N$ turns encountered by its ground shielding. The cross section of this simulated inductor is presented in the figure 2. Figure 3 shows the pattern of the square spiral coil with its ground shielding. After studying different spiral shape of the coil (square, circular and octagonal) and different number of turns, the design of figure 3 has given the better results [7]. 


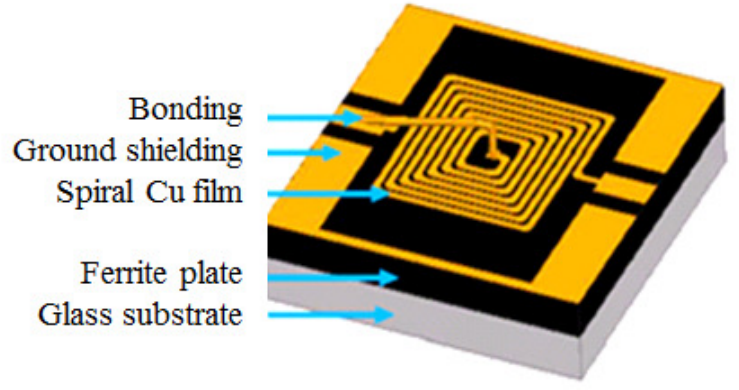

Figure 1: The spiral inductor structure with its magnetic core

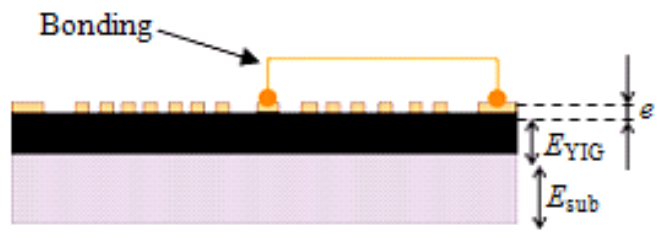

Figure 2: Cross section of the spiral inductor structure.

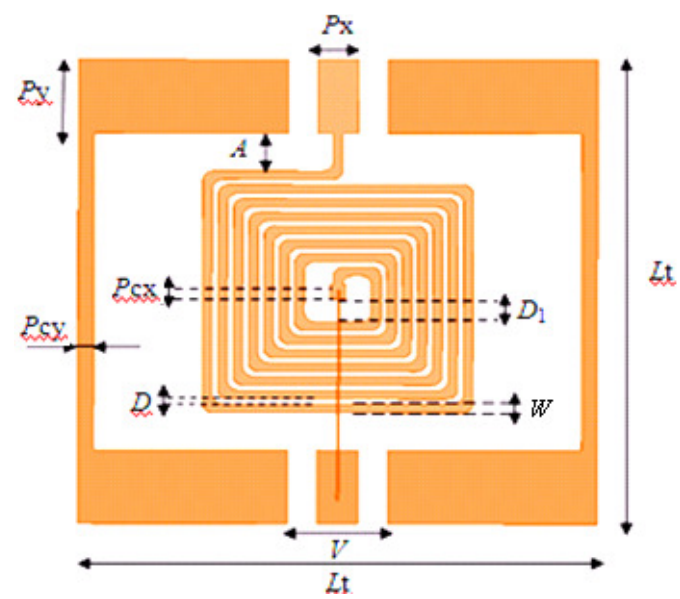

Figure 3: Pattern of the copper spiral coil with its ground shielding.

The geometrical magnitudes with their symbols are defined as follows:

- $W$ is the width of the spiral coil ribbon,

- $D$ is the distance between two successive turns,

- $D 1$ is the distance between the central terminal and the first turn,

- $P \mathrm{x}$ is the width of the external terminal,

- $P y$ is the length of the external terminal and the width of the ground shielding horizontal side,

- Pcx is the side of the central end of the spiral coil,

- Pcy is the width of the ground shielding vertical side,

- $V$ is the distance between the two parts of the ground shielding,

- $L t$ is the side of the ground shielding,

- $A$ is the distance between the external turn and the ground shielding horizontal side,

- $S$ is the area occupied by the spiral coil (without the ground shielding).
The values of all these geometrical magnitudes and those of figure 2 are summarized in the table 1 .

Table 1. Values of the geometrical parameters of the spiral inductor structure

\begin{tabular}{cc}
\hline Symbol & Size $(\mu \mathrm{m})$ \\
\hline$W$ & 125 \\
$D$ & 60 \\
$D 1$ & 400 \\
$P \mathrm{x}$ & 500 \\
$P \mathrm{y}$ & 1000 \\
$P \mathrm{cx}$ & 200 \\
$P \mathrm{cy}$ & 200 \\
$V$ & 1200 \\
$L t$ & 6270 \\
$A$ & 500 \\
$S$ & $3000 \mathrm{x} 3000$ \\
$N$ & 7 \\
$e$ & 5 \\
$E_{\mathrm{YIG}}$ & $50-1000$ \\
$E_{\text {sub }}$ & 1000 \\
\hline
\end{tabular}

\section{Technological steps of the spiral inductor fabrication}

The spiral inductor has been manufactured with the following technological steps (figure 4):

a) Preparation of the glass-ferrite substrate: The YIG wafer of $1 \mathrm{~mm}$ thickness is sawed at the same size of the glass substrate; it is then stuck on it to have the desired thickness. This plate is grinded by micromachining and it is then polished to obtain the required roughness measured by a profilometer.

b) Copper deposition: A film of copper ( $5 \mu \mathrm{m}$ thick) is deposited by RF sputtering technique on the whole YIG surface.

c) Deposition of a photo-resistive resin on the copper film by spin coating.

d) Insolation of the photoresist by using a mask having the same pattern of figure 3 .

e) Revelation of the exposed resin area.

f) Wet etching of the copper film.

g) Dissolution of the residual photoresist.

h) A gold thin film is deposited by electro-deposition in order to protect copper coil from oxidation

i) A bonding is performed to connect the central end of the spiral layer to the second external terminal

The photograph of the final manufactured inductor is showed in figure 5 . 


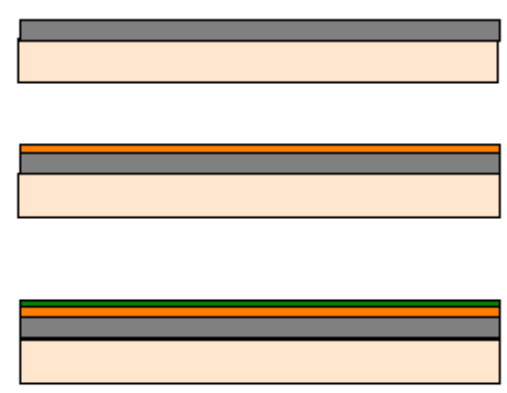

(a)

(b)

(c)
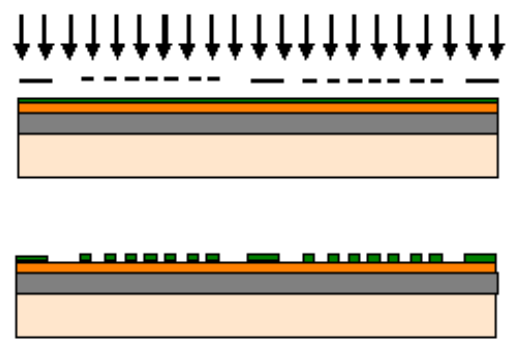

(e)
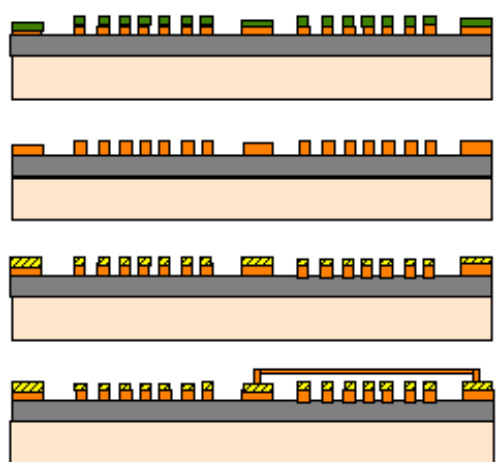

Figure 4: Manufacturing steps of the spiral inductor

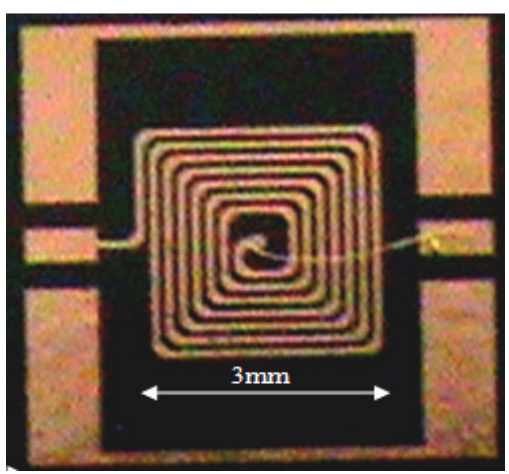

Figure 5: Photograph of the manufactured spiral inductor with its YIG core.

\section{Experimental and simulation results}

We present, firstly, the simulation results concerning the influence of the magnetic material on the inductance value of the spiral inductor prototype for different thicknesses. Secondly, a comparison between simulation and experimental frequency behaviors of the inductance $L$ and the series resistance $\mathrm{R}$ of the inductor will be presented.

\subsection{Influence of the magnetic material thickness}

The purpose of this section is to study the influence of the magnetic core on the inductance value of the integrated inductor. For this reason, we have done several simulations by varying the thickness of the magnetic material $\left(E_{\mathrm{YIG}}\right)$ from 0 (inductor without magnetic material) to $400 \mu \mathrm{m}$. At $100 \mathrm{MHz}$, the simulated inductance, obtained without magnetic material $\left(L_{\text {air }}\right)$ is about $100 \mathrm{nH}$. Figure 6 shows the evolution of the inductance with the thickness of the magnetic core. We can see easily the fast rise of the inductance in the thickness range from 0 to $100 \mu \mathrm{m}$. For $\mathrm{E}_{\mathrm{YIG}}$ equals to $100 \mu \mathrm{m}$, the inductance $L$ becomes practically 1.7 times $L_{\text {air. }}$ But from this thickness, $L$ varies slowly to reach 1.85 times $L_{\text {air }}$ for $\mathrm{E}_{\mathrm{YIG}}$ equals to $400 \mu \mathrm{m}$ and 1.95 (approximately twice $L_{\text {air }}$ ) for $1000 \mu \mathrm{m}$.

The variation of the inductance with the thickness of the YIG layer can be explained by the distribution of the field lines around the spiral coil [8]. This distribution is usually more concentrated near the coil and it will be more concentrated in the presence of a magnetic material in the vicinity of this structure and the field lines that are far from the coil will be less concentrated. This is why the value of inductance is not proportional to the thickness of the YIG wafer; it is not also proportional to its magnetic permeability. But for higher permeability, the inductance value may be doubled for a smaller thickness. To reach values more than twice $\mathrm{L}_{\mathrm{air}}$, we think introduce, in perspective, two magnetic wafers. For an ideal closed magnetic core, the inductance is multiplied by the permeability and reaches the value $\mu_{\mathrm{r}} . \mathrm{L}_{0}$.

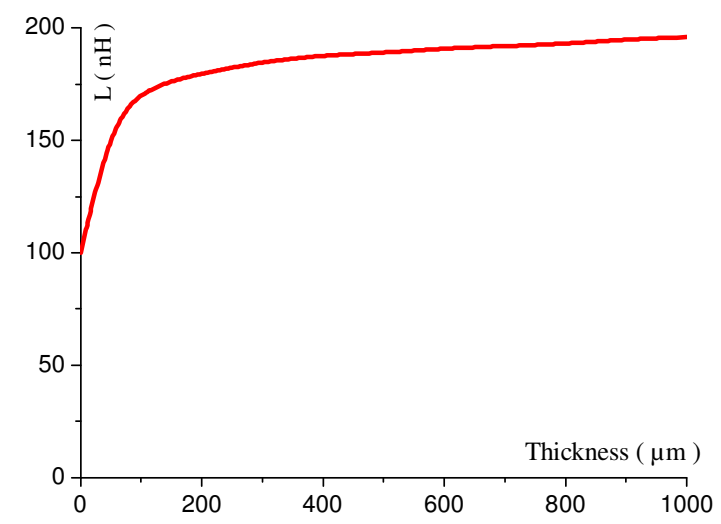

Figure 6: Variation of the inductance as a function of the magnetic material thickness.

\subsection{Frequency characterization of the spiral inductor}

The aim of this second section is to study the behavior of the inductor as a function of frequency. The measurements 
are performed using the vector network analyzer ZVA 67 for the frequency range from $10 \mathrm{MHz}$ up to $1.2 \mathrm{GHz}$. We have used the simulator ANSYS HFSS v.13 in order to compare the experimental results with the simulation one. In both cases, the thickness of the YIG wafer is $E_{\mathrm{YIG}}=$ $500 \mu \mathrm{m}$. For the simulation, we have chosen the following parameters for the YIG wafer: $\mu_{\mathrm{r}}=40$ for the relative permeability and $\varepsilon_{\mathrm{r}}=15$ for the relative permittivity (manufacturer's values). In the measurement as in simulation, we have used the results as $S$ parameters $\left(S_{\mathrm{ij}}\right)$. These parameters are then transformed into admittance parameters $\left(Y_{\mathrm{ij}}\right)$. The most suitable model is that of the figure 7 . In this model, $C_{\mathrm{fb}}$ is the coupling capacitance between the windings. By using this LRC model and the admittance parameters, we can extract the different elements of this model as follows [13]:

$$
\begin{aligned}
& Y=-Y_{12}=\frac{1}{\mathrm{R}+\mathrm{jL} \omega}+\mathrm{jC}_{\mathrm{fb}} \omega, \\
& \mathrm{R}=\frac{-\operatorname{Re}\left(\mathrm{Y}_{12}\right)}{\operatorname{Re}\left(\mathrm{Y}_{12}\right)^{2}+\left(\operatorname{Im}\left(\mathrm{Y}_{12}\right)+\mathrm{C}_{\mathrm{fb}} \omega\right)^{2}}, \\
& \mathrm{~L} \omega=\frac{\operatorname{Im}\left(\mathrm{Y}_{12}\right)+\mathrm{C}_{\mathrm{fb}} \omega}{\operatorname{Re}\left(\mathrm{Y}_{12}\right)^{2}+\left(\operatorname{Im}\left(\mathrm{Y}_{12}\right)+\mathrm{C}_{\mathrm{fb}} \omega\right)^{2}}, \\
& \mathrm{Q}=\frac{\operatorname{Im}\left(\mathrm{Y}_{12}\right)+\mathrm{Cfb}_{\mathrm{fb}}}{\operatorname{Re}\left(\mathrm{Y}_{12}\right)} \text {, } \\
& \mathrm{C}_{\mathrm{fb}}=\frac{1}{\left(2 \mathrm{f}_{\mathrm{o}} . \pi\right)^{2} \mathrm{~L}_{0}},
\end{aligned}
$$

$\mathbf{L}_{\mathbf{0}}$ is the inductance value at low frequency (initial inductance) and $f_{0}$ is the resonance frequency, obtained by plotting the imaginary part of $Y_{12}$. It corresponds to the frequency at which the curve of $\operatorname{Im}\left(Y_{12}\right)$ passes through zero.

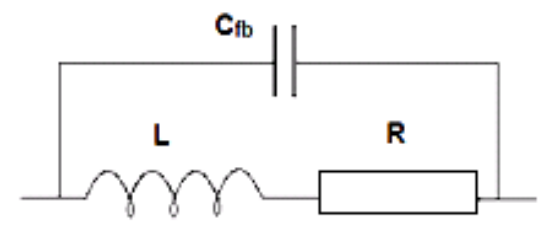

Figure 7: Series model of the integrated inductor with the coupling capacitance between windings.

\subsubsection{Frequency behavior of the inductance}

Figure 8 illustrates the simulated and the measured inductance as a function of frequency for $E_{\mathrm{YIG}}=500 \mu \mathrm{m}$. The curve corresponding to the experimental values shows that the inductance decreases with the increase of the frequency. This decrease is expected because in reality, the permeability of magnetic material decreases when the frequency increases [10]. But the simulated inductance remains practically constant versus frequency up to 0.9 $\mathrm{GHz}$. Because during the simulation, the value of the relative permeability is fixed to $\mu_{\mathrm{r}}=40$ for all frequencies from $10 \mathrm{MHz}$ up to $1.2 \mathrm{GHz}$. The gap between the two curves may be due to the calibration of the network analyzer, or to the fixed value of the magnetic permeability ( $\mu_{\mathrm{r}}=40$ ) during the simulation which is may be less than the real value. One can observe also, for the 2 curves, the sharp drop of the inductance at about $1 \mathrm{GHz}$. This frequency corresponds to the resonance of the structure.

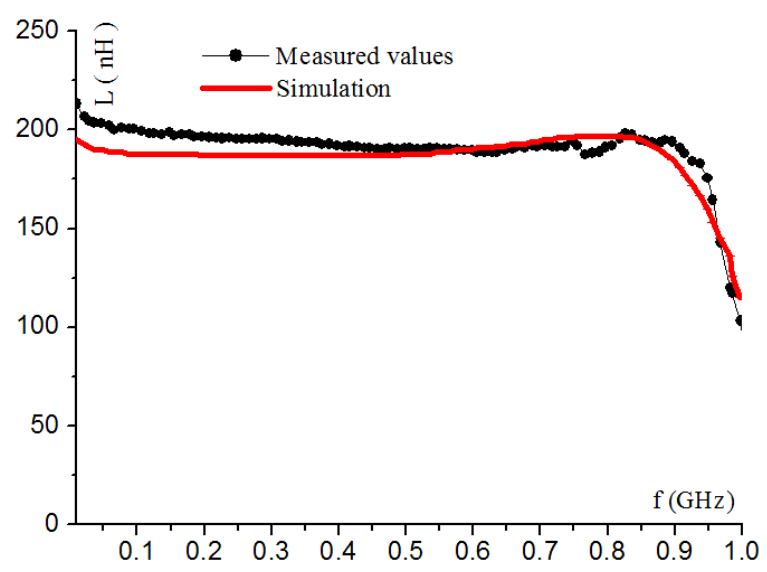

Figure 8: Evolution of the inductance versus frequency

\subsubsection{Frequency behavior of the resistance}

To study the evolution of the series resistance $R$ as a function of frequency, we have used HFSS software for the simulation, the LCR-meter HP 4284A working from $20 \mathrm{~Hz}$ to $1 \mathrm{MHz}$ for low frequency measurements and the vector network analyzer ZVA $67(10 \mathrm{MHz}-67 \mathrm{GHz})$ for high frequency.

The low frequency characterization was performed at 3 frequencies $(100 \mathrm{kHz}, 500 \mathrm{kHz}$ and $1 \mathrm{MHz})$. Table 2 shows the experimental results obtained for the series resistance of the manufactured prototype with a magnetic wafer of thickness $E_{\mathrm{YIG}}=500 \mu \mathrm{m}$. The simulation results correspond to the inductor of same dimensions.

Table 2: Resistance the spiral inductor as a function of frequency

\begin{tabular}{ccc}
\hline Frequency & Simulation & Measure \\
\hline $100 \mathrm{kHz}$ & $1.67 \Omega$ & $1.97 \Omega$ \\
$500 \mathrm{kHz}$ & $1.67 \Omega$ & $2.3 \Omega$ \\
$1 \mathrm{MHz}$ & $1.67 \Omega$ & $2.4 \Omega$ \\
\hline
\end{tabular}

At low frequency the simulated resistance of the spiral inductor is constant as showed in table 2. This resistance is independent of the frequency and of the magnetic core. It equals to the resistance of the copper spiral coil of figures 3 or 5. The calculated DC value of this resistance is $R_{D C}=$ $1.66 \Omega$, practically the same by comparing it with the simulated one $(1.67 \Omega)$. However, the measured resistance is higher than the simulated one. This could be explained by the fact that the thickness $e$ and the width $W$ of the copper ribbon are given constant for the simulation, but in reality these 2 magnitudes are not uniform because of the irregularity of the wet etching time. We can also say that the 
large experimental values of the resistance may be due to the oxidation of the copper ribbon surface.

The same manufactured inductor has been characterized at high frequency. In this range the coupling capacitance $C_{\mathrm{fb}}$ does not have any influence and the series resistance can be defined by the following equation:

$$
\mathrm{R}=\frac{-\operatorname{Re}\left(\mathrm{Y}_{12}\right)}{\operatorname{Re}\left(\mathrm{Y}_{12}\right)^{2}+\operatorname{Im}\left(\mathrm{Y} 12_{12}{ }^{2}\right.},
$$

The curves in Figure 9 illustrate the evolution of the simulated and measured series resistance of the integrated inductor as a function of frequency.

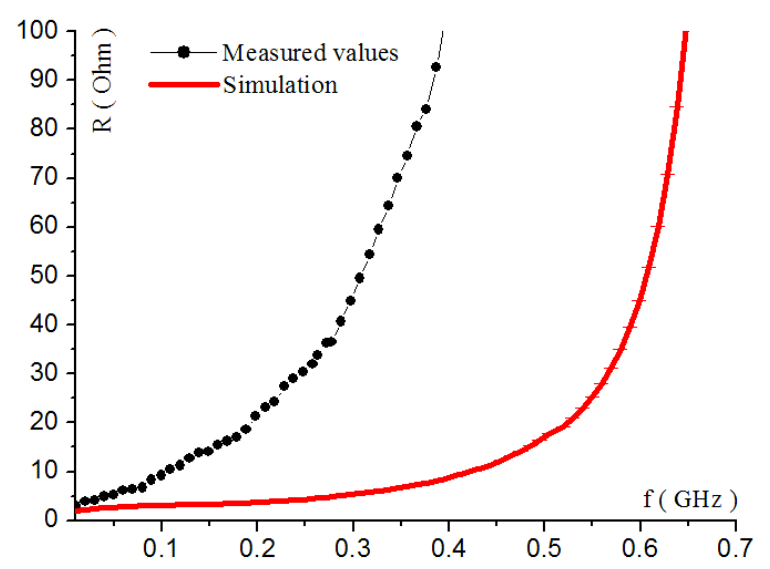

Figure 9: Resistance of the spiral inductor versus frequency

At $10 \mathrm{MHz}$, the resistance (3 $\Omega$ ) measured by the vector network analyzer is very close to that measured by the LCR-meter at $1 \mathrm{MHz}$. At these frequencies the skin and proximity effects are negligible. However, from $100 \mathrm{MHz}$, we notice that the measured resistance begins to increase rapidly due to the skin and the proximity effects [11-12]. These effects become important at high frequency. For example at $400 \mathrm{MHz}$, the resistance is multiplied by a factor of 21 by comparison with the measured value at $10 \mathrm{MHz}$.

However, there is a slow increasing of the simulated resistance up to $400 \mathrm{MHz}$ ( $R$ equals to $1.9 \Omega$ at $10 \mathrm{MHz}$ and $8.6 \Omega$ at $400 \mathrm{MHz}$ that corresponds to a ratio less than 5) because the proximity effects are not taken into account by HFSS and only skin effect are considered. This can explain the difference between simulation and measurement results. Other authors have mentioned the same phenomenon for inductors with FeHfN magnetic material. Anomalous losses have been observed that explain the decreasing of quality factor at high frequency [13-14].

The presence of the magnetic material, which helps to concentrate the field lines, permits to increase the magnetic field and consequently the inductance of the spiral inductor. However, the presence of thick magnetic core increase both magnetic filed and the proximity effects. When the thickness of the magnetic layer increases; the effects of induced currents in the copper spiral coil is greater. These effects cause the increase of the series resistance and consequently the decrease of quality factor.

\section{Conclusion}

To design of the integrated inductor with magnetic core we have used HFSS simulator. By changing the shape, the geometrical parameters and the number of turns, we have chosen the best configuration (square spiral inductor). To manufacture this inductor we have used different technique (micro-machining, RF sputtering, photolithography, electroplating, bonding ...). And to perform morphological and electromagnetic characterizations, we have used preferment equipment and software. We have found that the inductance increases by adding a magnetic layer to the spiral inductor; it could be doubled by increasing the thickness of the ferrite plate but this increase is limited by the proximity effects and the eddy currents at high frequency. The experimental results concerning the frequency dependence of the inductance and the series resistance of the manufactured integrated inductor are confirmed by HFSS simulations. In perspective, we will try to fabricate inductors with two magnetic layers to increase the performance of these components. The main purpose is to fabricate miniature integrated inductors for DC-DC converters.

\section{References}

[1] R. Melati, et al., Design of a new electrical model of a ferromagnetic planar inductor for its integration in a micro-converter, Mathematical and Computer 2011, doi:10.1016/jmcm.2011.06.014.

[2] J. Y. C. chang et al., IEEE Electron Device Lett.14, 246, 1993.

[3] J. N. Burghartz et al., IEEE Electron Device Lett.17, 428, 1996.

[4] Gyoung-Bum Kim, Seung-Yong Cha, Eun-Kyung Hyun,Young-Chai Jung, Yoon-Suk Choi, Jae-Sung Rieh, Seong-Rae Lee, and Sung-Woo Hwang, Integrated planar spiral inductors with $\mathrm{CoFe}$ and $\mathrm{NiFe}$ ferromagnetic layer, Microwave and optical technology letters, vol.50, No.3, 2008.

[5] C. Yang, F. Liu, T. Ren, L. Liu, H. Feng, A. Z. Wang, $\mathrm{H}$. Long, Fully integrated ferrite-based inductors for RF ICs, Sensors and Actuators A 130-131, 365-370, 2006.

[6] Ren Tianling, Yang Chen, Liu Litian, Wang A Z, and Zhang Xiao, Equivalent circuit analysis of an RF Integrated Inductor with Ferrite Thin- Film, Chinese Journal of Semiconductors, vol.27, No.3, 2006.

[7] D. Allassem, PhD Thesis, University of Saint Etienne, France, 2010

[8] G.E. Mechler, R.S. Girgis, Magnetic flux distributions in transformer core joints, IEEE Trans. On Power Delivery, Vol. 15, No. 1, 198, 2000.

[9] Yu Cao et al. Frequency -Independent Equivalent-circuit Model for on -chip spiral inductors, IEEE Journal of solid state circuits, Vol. 38, No.3, 2003.

[10] A. Kriga, D. Allassem, M. Soultan, J.P. Chatelon, A. Siblini, B. Allard, J.J. Rousseau, - Frequency characterization of thin soft magnetic material layers 
used in spiral inductors, J. of Magnetism and Magnetic Material, Vol. 324, No. 14, 2227-2232, 2012

[11] C.H. Ivan Lai and M. Fujishima, A New On-Chip Substrate-Coupled Inductor Model Implemented With Scalable Expressions"' IEEE Journal of solid state circuits, Vol. 41, No. 11, 2491-2499, 2006.

[12] Nam-Jin Oh and Sang-Gug Lee, A simple model parameter extraction methodology for on-chip spiral inductor, ETRI Journal, vol. 28, No. 1, 115-118, 2006.

[13] B. Viala, A.S. Royet, and S. Couderc, Investigation of Anomalous Losses in Thick $\mathrm{Cu}$ Ferromagnetic Spiral Inductors. IEEE Transactions on Magnetics, Vol. 41, No. 4, 3583-3585, 2005.

[14] A.S. Royet, B. Viala, S. Couderc, and B. Orlando, Investigation of proximity effects in ferromagnetic inductors with different topologies: modeling and solutions. Transactions of Magnetics Society of Japan, Vol.5, No.4, 144-145, 2005. 